
and Medicine

Vol. 11

\title{
TOWARDS A MATHEMATICAL THEORY OF COMPLEX BIOLOGICAL SYSTEMS
}




\section{SERIES IN MATHEMATICAL BIOLOGY AND MEDICINE}

\section{Series Editors: P. M. Auger and R. V. Jean}

Published

Vol. 1: Stochastic Models of Tumor Latency and Their Biostatistical Applications A. Yu. Yakovlev and A. D. Tsodikov

Vol. 2: Volterra-Hamilton Models in the Ecology and Evolution of Colonial Organisms P. L. Antonelli and R. H. Bradbury

Vol. 3: The Hierarchical Genome and Differentiation Waves Novel Unification of Development, Genetics and Evolution R. Gordon

Vol. 4: Symmetry in Plants Ed. by R. V. Jean and D. Barabe

Vol. 5: Computational Medicine, Public Health and Biotechnology: Building a Man in the Machine Proceedings of the First World Congress Vols. 1 \& $3-$ Ed. by M. Witten Vol. 2 - Ed. by D. Joan and M. Witten

Vol. 6: Advances in Mathematical Population Dynamics - Molecules, Cells and Man Proceedings of the 4th International Conference on Mathematical Population Dynamics Ed. by O. Arino, D. Axelrod and M. Kimmel

Vol. 7: Neuronal Information Processing From Biological Data to Modelling and Applications Ed. by G. Burdet, P. Combe and O. Parodi

Vol. 8: Advances in Bioinformatics and Its Applications Proceedings of the International Conference Ed. by M. He, G. Narasimhan and S. Petoukhov

Vol. 9: Handbook of Cancer Models with Applications Ed. by W.-Y. Tan and L. Hanin

Vol. 11: Towards a Mathematical Theory of Complex Biological Systems C. Bianca and N. Bellomo 


\section{Mathematical Biology} and Medicine

Vol. 11

\section{TOWARDS A MATHEMATICAL THEORY OF COMPLEX BIOLOGICAL SYSTEMS}

\section{Bianca • N Bellomo}

Politecnico di Torino, Italy 


\title{
Published by
}

World Scientific Publishing Co. Pte. Ltd.

5 Toh Tuck Link, Singapore 596224

USA office: 27 Warren Street, Suite 401-402, Hackensack, NJ 07601

UK office: 57 Shelton Street, Covent Garden, London WC2H 9HE

\author{
Library of Congress Cataloging-in-Publication Data \\ Bianca, Carlo. \\ Towards a mathematical theory of complex biological systems / by C. Bianca and N. Bellomo. \\ p. cm. -- (Series in mathematical biology and medicine ; v. 11) \\ Includes bibliographical references and index. \\ ISBN-13: 978-981-4340-53-3 (hardcover : alk. paper) \\ ISBN-10: 981-4340-53-7 (hardcover : alk. paper) \\ 1. Biomathematics. 2. Biological systems--Mathematical models. I. Bellomo, N. II. Title. \\ QH323.5.B4533 2011 \\ 570.15'118--dc22
}

2010043493

\section{British Library Cataloguing-in-Publication Data}

A catalogue record for this book is available from the British Library.

Copyright (C) 2011 by World Scientific Publishing Co. Pte. Ltd.

All rights reserved. This book, or parts thereof, may not be reproduced in any form or by any means, electronic or mechanical, including photocopying, recording or any information storage and retrieval system now known or to be invented, without written permission from the Publisher.

For photocopying of material in this volume, please pay a copying fee through the Copyright Clearance Center, Inc., 222 Rosewood Drive, Danvers, MA 01923, USA. In this case permission to photocopy is not required from the publisher.

Printed in Singapore. 


\section{Preface}

This monograph focuses on the ambitious aim of developing a mathematical theory of complex biological systems with special attention on the phenomena of ageing, degeneration, and repair of biological tissues under individual self-repair actions that may also take advantage of medical actions and therapies. The immune system plays an essential role in the competition against pathologies. Of course, the authors do not naively claim that such objective is effectively achieved. Simply some perspective ideas, and some preliminary steps, are brought to the attention of applied mathematicians.

The approach to the mathematical modeling of biological systems needs to tackle the additional difficulty generated by the peculiarity features of living matter. For instance the lack of invariance principles which are typical of systems pertaining to inert matter, the ability to express a strategy for individual wellbeing, heterogeneous behaviors, competition, proliferative and/or destructive actions, learning ability, evolution, and many others.

The presentation is organized in three parts with ten chapters, which have the aim of addressing applied mathematicians to research activity in the field of modeling complex biological systems viewed as living, and hence biological systems:

Part I is devoted to a phenomenological description of the aforementioned biological processes with the aim of capturing the characteristics that play a crucial role in the modeling process. A new systems biology approach is subsequently proposed, where the overall system is decomposed into subsystems on the basis of the biological functions that are expressed by each subsystem.

Part II presents the mathematical tools that are designed to achieve the aims of the monograph. These tools define a class of integro-differential equations that offer an appropriate underlying mathematical structure that can be used to derive models at the molecular and cellular scale. Moreover, mathematical methods to 
link the dynamics from the molecular to the cellular scale, and from this to that of tissues are studied in view of applications.

Part III deals with applications and perspectives. First, the modeling of two case studies is presented, namely the modeling of malignant keloid formation, and the derivation of chemotaxis models at the macroscopic scale from the underlying description at the cellular scale. Subsequently a critical analysis is proposed to understand how much work is still needed to achieve a mathematical theory of biological systems, and to identify the guidelines necessary to pursue this objective. In more details, the sequential steps of the mathematical approach are as follows: i) Modeling biological systems as a large, heterogeneous, multiscale complex system;

ii) Integration of the concept of functional subsystems to reduce the complexity of large living systems within the approach of systems biology;

iii) Development of the mathematical kinetic and stochastic game theory for active particles;

iv) Modeling interactions at the molecular and cellular scale and application of the theoretical approach to some case studies;

v) Development of a multiscale analysis and modeling from genes to tissues.

The final aim of this monograph is to provide a new conceptual background for applied mathematicians involved in the challenging research field of mathematics of living systems. The authors trust that the validity of the methodological will not be limited to the specific cases treated in the monograph, but it will provide the background for a large variety of problems generated by the interdisciplinary approach of mathematics and biology.

The authors are indebted to the Partners of the Project RESOLVE of the European Union. Their contribution to understand complex biological phenomena and to their modeling by an interdisciplinary mathematical-biological approach has been a precious gift.

Torino,

Carlo Bianca

September 2010

Nicola Bellomo 


\section{Acknowledgments}

The authors acknowledge the support by the European Union FP7 Health Research Grant number FP7-HEALTH-F4-2008-202047-RESOLVE, the support by the FIRB project RBID08PP3J-Metodi matematici e relativi strumenti per la modellizzazione e la simulazione della formazione di tumori, competizione con il sistema immunitario, e conseguenti suggerimenti terapeutici, and the Compagnia di SanPaolo, Torino, Italy.

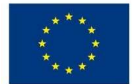

Funded by the European Commission

FP7 Health Research

Grant number FP7-HEALTH-F4-2008-202047 
This page intentionally left blank 


\section{Contents}

Preface $\quad$ v

Acknowledgments $\quad$ vii

List of Figures $\quad$ xiii

List of Tables $\quad$ xvii

1. Looking for a Mathematical Theory of Biological Systems 1

1.1 Introduction . . . . . . . . . . . . . . . . . . . 1

1.2 On the Concept of Mathematical Theory . . . . . . . . . . 2

1.3 Plan of the Monograph . . . . . . . . . . . . . 3

2. On the Complexity of Biological Systems 7

2.1 Ten Common Features of Living Systems . . . . . . . . . . 7

2.2 Some Introductory Concepts of Systems Biology . . . . . . . . . 10

2.3 Reducing Complexity . . . . . . . . . . . . . . . . . 13

\section{Immune System, Wound Healing Process, and System Biol-} ogy

3. The Immune System: A Phenomenological Overview 17

3.1 Introduction . . . . . . . . . . . . . . . . . . 17

3.2 Bacteria and Viruses . . . . . . . . . . . . . . 18

3.3 The Immune System Components … . . . . . . . . . . . . . 19

3.3.1 The Lymphatic System _ . . . . . . . . . . . . . . . 19

3.3.2 The White Blood Cells . . . . . . . . . . . . . 21 
3.3.3 Antibodies and Hormones . . . . . . . . . . . . . . . 24

3.4 The Immune Response . . . . . . . . . . . . . . . . . . . . . . 25

3.4.1 Innate Immunity . . . . . . . . . . . . . . . . 26

3.4 .2 Adaptive Immunity . . . . . . . . . . . . . . . . . . . . . . . . 29

3.5 Immune System Diseases . . . . . . . . . . . . . . . . . . 32

3.6 Critical Analysis . . . . . . . . . . . . . . . 35

4. Wound Healing Process and Organ Repair 37

4.1 Introduction . . . . . . . . . . . . . . . . . 37

4.2 Genes and Mutations . . . . . . . . . . . . . . 38

4.3 The Phases of Wound Healing . . . . . . . . . . . . . 43

4.3.1 Hemostasis Phase . . . . . . . . . . . . . . . 44

4.3.2 Inflammation Phase . . . . . . . . . . . . . . 47

4.3.3 Proliferation Phase . . . . . . . . . . . . . . . . . . 48

4.3.4 Maturation or Remodeling Phase . . . . . . . . . . . . 49

4.4 The Fibrosis Disease . . . . . . . . . . . . . . . . . 50

4.5 Critical Analysis . . . . . . . . . . . . . . . . . 54

5. From Levels of Biological Organization to System Biology 55

5.1 Introduction . . . . . . . . . . . . . . 55

5.2 From Scaling to Mathematical Structures _ . . . . . . . . . 56

5.3 Guidelines to the Modeling Approach . . . . . . . . . . 60

\section{$\begin{array}{ll}\text { Mathematical Tools } & 65\end{array}$}

6. Mathematical Tools and Structures 67

6.1 Introduction . . . . . . . . . . . . . . 67

6.2 Mathematical Frameworks of the Kinetic Theory of Active Particles . . . . . . . . . . . . . . . . . . 68

6.3 Guidelines Towards Modeling at the Molecular and Cellular Scales . . . . . . . . . . . . . . . . . 78

6.4 Additional Analysis Looking at the Immune Competition . . . . 80

6.5 Critical Analysis . . . . . . . . . . . . . . . 85

7. Multiscale Modeling: Linking Molecular, Cellular, and Tissues Scales

7.1 Introduction . . . . . . . . . . . . . . . . . 89 
7.2 On the Phenomenological Derivation of Macroscopic Tissue Models ... . . . . . . . . . . . . . . . . . 91

7.3 Cellular-Tissue Scale Modeling of Closed Systems . . . . . . . 94

7.3.1 Asymptotic Methods for a Single Subsystem . . . . . . 95

7.3.2 Asymptotic Methods for Binary Mixtures of Subsystems 99

7.4 Cellular-Tissue Scale Modeling of Open Systems . . . . . . . . 108

7.5 On the Molecular-Cellular Scale Modeling . . . . . . . . . . . 111

7.6 Critical Analysis .................. 113

\section{Applications and Research Perspectives}

8. A Model for Malign Keloid Formation and Immune System Competition 119

8.1 Introduction . . . . . . . . . . . . . . . . . . . 119

8.2 The Mathematical Model . . . . . . . . . . . . . . . 121

8.3 Simulations and Emerging Behaviors . . . . . . . . . . . 131

8.3.1 Sensitivity Analysis of the Progression Rate $\alpha$. . . . 132

8.3.2 Sensitivity Analysis of the Proliferation Rate $\beta_{I}$. . . . 144

8.3.3 Sensitivity Analysis of the Initial Distributions . . . . . 147

8.4 Critical Analysis and Perspectives . . . . . . . . . . . . 154

9. Macroscopic Models of Chemotaxis by KTAP Asymptotic Methods 157

9.1 Introduction . . . . . . . . . . . . . . . . . . 157

9.2 Linear Turning Kernels: Relaxation Models . . . . . . . . . . . 159

9.2.1 The Case of a Single Subsystem . . . . . . . . . . 160

9.2.2 The Case of a Binary Mixture of Subsystems . . . . . . 162

9.3 Cellular-Tissue Scale Models of Chemotaxis . . . . . . . . . . 163

9.3.1 Classical Keller-Segel Type Models . . . . . . . . . . . 165

9.3.2 Optimal Drift Following the Chemoattractant . . . . . . 165

9.3.3 Nonlinear Flux-Limited Model by the Mixed Scalings . 166

9.4 Critical Analysis . . . . . . . . . . . . . . 168

10. Looking Ahead 171

10.1 Introduction . . . . . . . . . . . . . . . . 171

10.2 Some Challenges for Applied Mathematicians and Biologists . . 172

10.3 How Far is the Mathematical Theory for Biological Systems . . 173

10.4 Closure .................... 177

Appendix A Mathematical Modeling of Space and Velocity- 
Dependent Systems

A.1 Introduction . . . . . . . . . . . . . . . . 179

A.2 Mathematical Tools for Homogeneous Activity Systems . . . 179

A.3 Mathematical Tools for Heterogeneous Activity Systems . . . . 182

$\begin{array}{ll}\text { Glossary } & 187\end{array}$

$\begin{array}{ll}\text { Bibliography } & 195\end{array}$

$\begin{array}{ll}\text { Index } & 205\end{array}$ 


\section{List of Figures}

2.1 A representation of the process of differentiation. . . . . . . . 10

3.1 The recognition process of the adaptive immune system: Under antigenic stimulus, B-lymphocytes interact with macrophages and $\mathrm{T}$ helper cells, and proliferate, differentiate into antibody-secreting plasma cells. The plasma cells synthesize large amounts of immunoglobulins (antibodies) which will react stereochemically with the stimulating antigen. The secreted antibody binds to the antigen and in some way leads to its neutralization or elimination from the body. B-lymphocytes and T-lymphocytes also develop into clones of identical reactive cells called memory B-cells and T-cells. . . . . . . . 30

4.1 The structure of a gene and its products. . . . . . . . . . . . . 40

4.2 The phases of cutaneous wound healing. . . . . . . . . . . . . . . 45

4.3 Sequence of phases of normal wound healing. . . . . . . . . . . 46

5.1 Dynamics of gene expression inside a cell. . . . . . . . . . . . 57

5.2 Chemical substances, attract blood vessels, with genesis of capillary sprouts from existing vasculature to feed the tumor. . . . . . . . . . 61

6.1 Long range interactions . . . . . . . . . . . . . 71

6.2 Destructive interactions . . . . . . . . . . . . . . 71

6.3 Proliferative interactions . . . . . . . . . . . . 72

6.4 Interactions with transition into a new subsystem $\ldots \ldots \ldots . . .74$

8.1 The time evolution of the densities of KFc (solid line) and of Mc (dashed line) for $\alpha=0.3$. The low magnitude of the progression rate never allows the number of $\mathrm{Mc}$ to overcome the number of $\mathrm{KFc} \quad \ldots 133$ 
8.2 The distribution function of the AV for $\alpha=0.1$ (top panel) and $\alpha=$ 0.3 (bottom panel).

8.3 The distribution function of the KFc for $\alpha=0.1$ (top panel) and $\alpha=$ 0.3 (bottom panel).

8.4 The distribution function of the Mc for $\alpha=0.1$ (top panel) and $\alpha=$ 0.3 (bottom panel).

8.5 The time evolution of the density of the ISc for $\alpha=0.5$. The ISc proliferate before reaching a plateau.

8.6 The time evolution of the densities of $\mathrm{KFc}$ (solid line) and of $\mathrm{Mc}$ (dashed line) for $\alpha=0.4$ (top panel) and $\alpha=0.5$ (bottom panel).

8.7 The distribution function of the $\mathrm{AV}$ for $\alpha=0.4$ (top panel) and $\alpha=$ 0.5 (bottom panel).

8.8 The distribution function of the KFc for $\alpha=0.4$ (top panel) and $\alpha=$ 0.5 (left panel).

8.9 The distribution function of the Mc for $\alpha=0.4$ (top panel) and $\alpha=$ 0.5 (bottom panel).

8.10 Time evolution of the densities of the $\mathrm{KFc}$ (solid line) and the Mc (dashed line) at the top panel and of the NFc (dotted line), AV (circle line), and ISc (square line) at the bottom panel $(\alpha=0.8)$.

8.11 Distribution functions of the Mc (top panel) and AV (bottom panel) for $\alpha=0.8$.

8.12 The distribution functions of $\mathrm{AV}, \mathrm{KFc}$, and $\mathrm{Mc}$ at times $t=3$ (top panel) and $t=8$ (bottom panel) for $\beta_{I}=0 \ldots \ldots \ldots \ldots . \ldots 144$

8.13 The time evolution of the number density of the ISc for $\beta_{I}=0 \ldots \ldots 145$

8.14 Distribution function of the NFc (top panel) and KFc (bottom panel) for $\beta_{I}=0.8 \ldots \ldots \ldots \ldots \ldots \ldots \ldots \ldots \ldots \ldots \ldots \ldots$

8.15 The distributions $g_{1}(u), g_{10}(u)$, and $g_{15}(u)$.

8.16 The time evolution of the number density of the KFc (solid line) and Isc (square line) in the top panel and the distribution function of KFc in the bottom panel when $f_{1}(0, u)=g_{10}(u), f_{2}(0, u)=g_{0}(u)$, and $f_{5}(0, u)=g_{1}(u)$.

8.17 The time evolution, when $f_{1}(0, u)=g_{15}(u), f_{2}(0, u)=g_{10}(u)$, and $f_{5}(0, u)=g_{1}(u)$, of the density of NFc (dotted line) and AV (circle line) in the top panel, and of the density of the KFc (solid line) and Mc (dashed line) in the bottom panel.

8.18 The time evolution of the density of NFc (dotted line) and of the AV (square line) for $f_{1}(0, u)=f_{2}(0, u)=g_{10}(u)$ and $f_{5}(0, u)=g_{15}(u) \ldots 151$

8.19 The distribution function of the KFc (top panel) and Mc (bottom panel) for $f_{1}(0, u)=f_{2}(0, u)=g_{10}(u)$ and $f_{5}(0, u)=g_{15}(u)$. 
8.20 The time evolution of the densities of NFc (dotted line), AV (circle line), and ISc (square line) in the top panel, and of the KFc (solid line) and Mc (dashed line) in the bottom panel, for $f_{1}(0, u)=g_{10}(u)$, $f_{2}(0, u)=g_{15}(u)$, and $f_{5}(0, u)=g_{1}(u) . \ldots \ldots \ldots \ldots$

9.1 From genes to tissues. . . . . . . . . . . . . . . . . . . . . . . . . 169 
This page intentionally left blank 


\section{List of Tables}

3.1 The fundamental differences between the innate and adaptive immune system. . . . . . . . . . . . . . . 26

8.1 Functional subsystems, interaction terms, and related parameters. . . . 130

8.2 The results of the simulations on the sensitivity analysis of the progression rate $\alpha$ of the KFc (first block corresponding to $\alpha=$ $0.2,0.5,0.8$ ), and of the proliferation rate $\beta_{I}$ of the ISc (second block

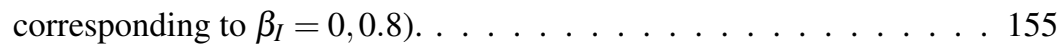

8.3 The results of the simulations on the sensitivity analysis of the initial number density $n_{i}(0)$, for $i \in\{1,2,5\}$, assuming $n_{4}(0)=n_{5}(0)=0 . \quad 156$ 